\title{
TiNi Alloy as a Highly Sensitive Bolometer Material
}

\author{
Pavel Vybornov ${ }^{1,2, *}$ and Viktor Erofeev ${ }^{1}$ \\ 'Institute of Monitoring of Climatic and Ecological Systems of Siberian Branch of the Russian \\ Academy of Sciences, 10/3, Academichesky Ave., Tomsk, 634055, Russia \\ ${ }^{2}$ Siberian Physical-Technical Institute of Tomsk State University, \\ 1, Novosobornaya Square, Tomsk 634050, Russia
}

(Received April 18, 2013; accepted July 25, 2013)

Key words: $\quad$ titanium nickelide, thin film, bolometer, responsivity

We have conducted a comparative study of the characteristics of thin films constructed from nickel and alloy of titanium nickelide exposed to optical radiation. We observed a sevenfold superiority in the sensitivity of the TiNi samples over that of the $\mathrm{Ni}$ samples under identical experimental conditions when the samples were of comparable overall dimension. A hypothesis pertaining to the advantage of using titanium nickelide (as opposed to other technologically applicable metals and alloys) for the sensitive element in a bolometer has been confirmed. We have determined the dependence of relevant parameters of thin film titanium nickelide on the characteristics of preliminary thermomechanical processing of the alloy.

\section{Introduction}

Bolometers are thermal radiation receivers, whose operation is based on the change in electrical resistance that occurs in the sensitive element when the temperature of that element changes in response to incident radiant flux. ${ }^{(1)}$

Depending on the material of the sensitive element, there is a difference between semiconducting and metal bolometers. The metals $\mathrm{Pt}, \mathrm{Au}, \mathrm{Ni}, \mathrm{Nb}$, and $\mathrm{Ti}$ are widely used for the sensitive element in metal bolometers. Despite the fact that metal bolometers have a simple design, are highly effective, and are characterized by high optical damage threshold, stability of parameters and a low level of noise, their major shortcoming is low sensitivity.

Single-element and matrix bolometers are used in the mid- and far-IR, and also in the terahertz band to solve a wide range of tasks: from the measurement of radiation power to the visualization of objects. ${ }^{(2,3)}$ Nevertheless, heightened interest remains for improving the processing of basic materials and increasing sensitivity, reducing noise, widening their sensitivity bandwidth and improving their performance through the optimization of mass-dimensional indexes and packaging. ${ }^{(4)}$

${ }^{*}$ Corresponding author: e-mail: yavay@sibmail.com 
Titanium nickelide alloys are well known for the property of doing work during the transformation from a single-phase low-temperature (high-temperature) state to a twophase state (shape memory effect) under the influence of an optical emission. ${ }^{(5)}$ Tests have shown that these materials are highly sensitive to small changes in temperature. This fact provides motivation for studying the feasibility of using these alloys in the creation of highly sensitive bolometers.

\section{Materials and Methods}

\subsection{Background theory}

Responsivity is one of the key parameters for optical radiation receivers. For bolometers, it is given by:

$$
S=(\delta V / P)=(I \delta R / P)
$$

where $I$ is the current through the bolometer, $\delta V$ and $\delta R$ are the changes in voltage drop and electrical resistance, respectively, of the element (thin metal film) of the bolometer that is sensitive to the incident radiant flux with power level $P$. In turn, the change in electrical resistance of the film is given by:

$$
\delta R=R_{\mathrm{i}} \alpha \Delta T
$$

where $R_{\mathrm{i}}$ is the electrical resistance of the film at the initial temperature, $\Delta T$ is the change in temperature, and $\alpha$ is the coefficient of thermal resistance (TCR) of the metal from which the film is produced. Neglecting the self-heating of the film, ${ }^{(6)}$ the increase in temperature is determined by: ${ }^{(7,8)}$

$$
\Delta T=\frac{\eta P}{G}=\frac{\eta P \tau}{H}
$$

where $P$ and $\eta$ are the power and absorption coefficient of optical radiation, respectively, $\tau$ is the time constant of the film, $G$ is the thermal conductance, and $H$ is the thermal mass. Substituting $\Delta T$ and geometrical dimensions of the films in eq. (2), we have:

$$
\delta R=\rho_{\mathrm{i}} \frac{l}{h w} \alpha \frac{\eta P \tau}{c_{\mathrm{P}} \rho l h w},
$$

where $\rho_{\mathrm{i}}$ is the electrical resistance of the film at the initial temperature, $l, h$, and $w$ are the length, width and thickness, and $\rho$ and $c_{\mathrm{P}}$ are the density and specific heat capacity of the metal from which the film is produced, respectively. For comparison purposes, the ratio of the responsivities of two bolometers (with films of differing metals or alloys but having the same geometric dimensions and subjected to identical experimental conditions) will be as follows:

$$
\frac{S_{1}}{S_{2}}=\frac{\rho_{\mathrm{i} 1} \alpha_{1} \tau_{1}}{\rho_{\mathrm{i} 2} \alpha_{2} \tau_{2}} \frac{c_{\mathrm{P} 2 \rho_{2}}}{c_{\mathrm{P} 1} \rho_{1}}
$$


It is apparent from eq. (5) that the responsivity of the bolometer depends on a combination of physical properties of the metals (or alloys) used in producing the thin films. Table 1 provides the reference data at the temperature of $20{ }^{\circ} \mathrm{C}$ for TiNi and metals widely used in bolometers along with the calculated ratio of their comparative responsivities (neglecting the self-heating of the films and heat losses, i.e., ignoring the ratio of $\left.\tau_{1} / \tau_{2}\right)$.

Note that for the films produced from chromium $(\mathrm{Cr})$, tin $(\mathrm{Sn})$, lead $(\mathrm{Pb})$, hafnium $(\mathrm{Hf})$ and zirconium ( $\mathrm{Zr}$ ), the $S_{\mathrm{TiNi}} / \mathrm{S}_{\text {metal }}$ ratios are equal to 8, 6.8, 5.9, 3.7, and 2.8, respectively. For the rest of the technologically applicable metals and alloys, it is greater than 10 . The values of TCR for Ni and TiNi (single-phase low-temperature state) were obtained by measurement using the samples of this study.

As can be seen from the calculations (Table 1), equiatomic titanium nickelide has the best combination of physical properties to create highly sensitive metal bolometers. ${ }^{(9)}$ In this work, a comparative study of the $\mathrm{Ni}$ and equiatomic TiNi thin films after exposure to optical radiation is carried out.

\subsection{Experiments}

Thin films of titanium nickelide are produced from stock sheet subjected to the standard processing steps required to obtain a plate of $0.5 \mathrm{~mm}$ thickness. The alloy is refined by induction heating in a protective atmosphere formed using original homemade vacuum equipment and neutral gases (argon). The TiNi-1 and TiNi-2 films were produced using different technologies: the samples of $\mathrm{Ni}$ and $\mathrm{TiNi}-1$ were produced by repeated rolling of the stock sheet with intermediate annealing and subsequent dissolution in acid; TiNi-2 was produced by mechanical polishing. For comparison, we have chosen a Ni film that has one of the highest TCR values among the metals. All samples were covered with an identical layer of black gel ink. The resultant absorption coefficient was $0.43 \pm 0.02$.

The average geometric dimensions of each film sample are shown in Table 2, where $r$ is the distance between the parallel soldered voltage leads.

Table 1

Comparative characteristics of metals and TiNi.

\begin{tabular}{lcccccc}
\hline Parameters & $\mathrm{Pt}$ & $\mathrm{Au}$ & $\mathrm{Ni}$ & $\mathrm{Ti}$ & $\mathrm{Nb}$ & $\mathrm{TiNi}$ \\
\hline$\alpha\left(10^{-3} \cdot \mathrm{K}^{-1}\right)$ & 3.85 & 3.96 & 7.4 & 3.5 & 3.95 & 3.2 \\
$\rho(\mathrm{g} / \mathrm{cm} 3)$ & 21.45 & 19.3 & 8.9 & 4.5 & 8.6 & 6.45 \\
$\rho_{\mathrm{i}}(\mu \Omega \cdot \mathrm{m})$ & 0.105 & 0.022 & 0.069 & 0.42 & 0.15 & 1.1 \\
$c_{\mathrm{P}}(\mathrm{J} / \mathrm{kg} \cdot \mathrm{K})$ & 133 & 129 & 445 & 524 & 265 & 200 \\
$S_{\text {TiNi }} / S_{\text {metal }}$ & 19 & 78 & 21 & 4.4 & 10.5 & 1 \\
\hline
\end{tabular}

Table 2

Dimensions of metal films.

\begin{tabular}{lccl}
\hline Parameters & Ni & TiNi-1 & TiNi-2 \\
\hline$l(\mathrm{~mm})$ & 2.08 & 2.05 & 2 \\
$r(\mathrm{~mm})$ & 1.15 & 0.65 & 0.7 \\
$h(\mathrm{~mm})$ & 1.38 & 1.2 & 1.35 \\
$w(\mu \mathrm{m})$ & 38 & 47 & 43 \\
\hline
\end{tabular}


The linear dimensions $l, h$, and $r$ were measured using a microscope Axio Lab.A1 (Carl Zeiss) with an accuracy of $\pm 5 \mu \mathrm{m}$. The thickness $w$ was determined using an R375 (Mitutoyo) digital micrometer with an error of $\pm 1 \mu \mathrm{m}$. The electrical resistivity of each sample was checked using the formula:

$$
\rho_{\mathrm{i}}=\frac{R_{\mathrm{i}} w h}{r} .
$$

The value of $R_{\mathrm{i}}$ was taken from the measurement results of the temperature dependence for the electrical resistivity of thin films (Fig. 1). Measurements were performed in a liquid thermostat Haake DC-30 (Thermo Electron Corporation) with a temperature stability $\pm 0.1^{\circ} \mathrm{C}$. The electrical resistance of the samples was measured using a Milliohmmeter E6-25 (All-Bright Technology) with a basic accuracy of $\pm 0.03 \%$. The values that were obtained for $\rho_{\mathrm{i}}$ (for the temperature of $20{ }^{\circ} \mathrm{C}$ within $\pm 4.5 \%$ ) corresponded to the reference data given in Table 1.

The changes in electrical resistance and time constant of the thin films during the action of laser radiation were measured using the experimental setup shown in Fig. 2.

The experimental setup included: a DC power supply HY3003D-2 (Mastech) -1 ; computers -2; oscilloscope WaveJet 322 (LeCroy) -3; amperemeter A based on the multimeter U1251B (Agilent Technologies) with a measurement error not exceeding $\pm 0.4 \%$; metal film sample B; instrumentation amplifier D1; operational amplifier D2; current limiter $\mathrm{R}$; load resistance $\mathrm{R}_{\mathrm{L}}$; switch $\mathrm{S}$; voltmeter $\mathrm{V}$ based on the multimeter UT71C (Uni-Trend Technology) with a measurement error of less than $\pm 0.04 \%$. The letter «O» in Fig. 2 denotes the focusing area of the laser beam.

The experimental method was as follows. The Ni, TiNi-1 and TiNi-2 films, coated by an optical radiation absorption layer and soldered (on the opposite side) with leads from a copper wire of $120 \mu \mathrm{m}$ diameter, were placed in a protective case. The laser
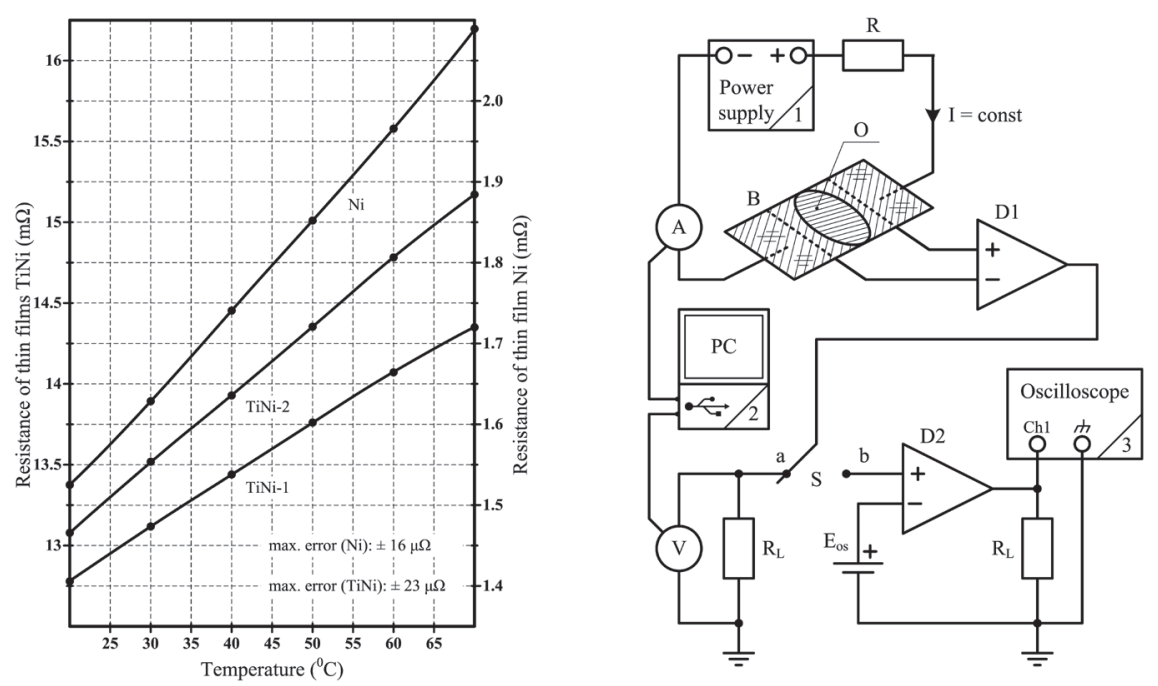

Fig. 1 (left). Electrical resistivity versus temperature for the samples of Ni, TiNi-1 and TiNi-2 thin films.

Fig. 2 (right). Experimental setup. 
(with a lens) was mounted on the lid of the protective case with the option of horizontal movement in any direction.

Initially, the switch S was set to "a" position. Voltage, applied to the output of a DC power supply, produced $1 \mathrm{~A}$ of current that flowed through the film under investigation. The voltage drop in the film was amplified and then recorded using a voltmeter. For the Ni film, the amplification coefficient of the instrumentation amplifier D1 was 98.8 \pm 0.2 ; for the other films, $19.31 \pm 0.06$. The data of the voltmeter and amperemeter were recorded by a computer via a USB interface with a speed of one measurement per second. After the stabilization of the film's electrical resistivity, the laser was turned on with a wavelength of $650 \mathrm{~nm}$. The laser beam was focused on the "O", as shown in Fig. 2. The laser was operating in a pulsed-periodic mode with a pulse duration of $10 \mathrm{~s}$ and a pulse ratio of 2 . The rise and fall times of optical pulses were less than $20 \mu \mathrm{s}$. The experimental data were collected for 20 full periods of the laser operation.

Measuring the time constant of the thin films required the introduction of an additional stage for the operational amplifier. For this purpose, the switch $\mathrm{S}$ was moved to "b" position. The operational amplifier D2 was set to zero by means of the $\mathrm{E}_{\mathrm{OS}}$ bias voltage. The change in load voltage after turning on the laser for 10 full periods of the operation was recorded by an oscilloscope in self-record mode. The amplification coefficient of the operational amplifier D2 did not change throughout all the experiments and was equal to 10 . The laser power was recorded by a high-sensitivity thermopile sensor 818P-001-12 (Newport Corporation) before and after each cycle of measurements with an accuracy of $\pm 2.5 \%$.

Moreover, using the experimental setup and a liquid thermostat, TCR of the thin film samples was determined in the temperature range of $20-70^{\circ} \mathrm{C}: \alpha_{\mathrm{Ni}}=7.40 \times 10^{-3} \pm 0.16 \times 10^{-3} \mathrm{~K}^{-1}$; $\alpha_{\mathrm{TiNi}-1}=2.49 \times 10^{-3} \pm 0.07 \times 10^{-3} \mathrm{~K}^{-1} ; \alpha_{\mathrm{TiNi}-2}=3.22 \times 10^{-3} \pm 0.08 \times 10^{-3} \mathrm{~K}^{-1}$. Measurements of TCR for the Ni and TiNi-2 films corresponded to the values of TCR for their respective stock sheets.

\section{Results and Discussion}

The responsivities of the thin film samples were calculated on the basis of empirical measurement in accordance with the following formula:

$$
S=\frac{\delta V}{P+\delta V I}=\frac{\frac{\left(V_{\mathrm{a}}-V_{\mathrm{i}}\right)}{k}}{P+\frac{\left(V_{\mathrm{a}}-V_{\mathrm{i}}\right)}{k} I},
$$

where $V_{\mathrm{i}}$ is the average measured voltage registered from the sample before the action of laser radiation, $V_{\mathrm{a}}$ is the average measured voltage registered from the sample under the action of laser radiation, $I$ is the average value of the measured current before and under the action of laser radiation, $k$ is the amplification coefficient of the circuit, $P$ is the laser radiation power and $\delta V I$ is the self-heating power of the thin films. Note that in each experiment, variations of current $I$ did not exceed $0.01 \mathrm{~A}$, as well as the variations of voltages $V_{\mathrm{i}}$ and $V_{\mathrm{a}}$ of $0.3 \mathrm{mV}$ in relation to average magnitudes. The experimental data and calculated responsivity of the thin film samples are given in Table 3.

It was established that for all thin films the influence of the self-heating powers $\delta V I$ on their responsivities did not exceed 1\%. Besides, the increase in P led to an increase in 
Table 3

Experimental and calculated data.

\begin{tabular}{clccc}
\hline \multicolumn{2}{c}{ Parameter } & $\mathrm{Ni}$ & $\mathrm{NiTi}-1$ & $\mathrm{NiTi}-2$ \\
\hline $28 \mathrm{~mW}$ & $I(\mathrm{~A})$ & 1.0595 & 1.0613 & 1.0574 \\
& $V_{\mathrm{i}}(\mathrm{mV})$ & 158.67 & 270.14 & 275.26 \\
& $V_{\mathrm{a}}(\mathrm{mV})$ & 167.85 & 278.24 & 291.61 \\
& $S(\mathrm{mV} / \mathrm{W})$ & 3.31 & 14.75 & 29.32 \\
\hline $58 \mathrm{~mW}$ & $I(\mathrm{~A})$ & 1.0608 & 1.0621 & 1.0583 \\
& $V_{\mathrm{i}}(\mathrm{mV})$ & 159.31 & 270.65 & 275.76 \\
& $V_{\mathrm{a}}(\mathrm{mV})$ & 181.32 & 287.51 & 309.93 \\
& $\tau(\mathrm{ms})$ & 325 & 325 & 290 \\
& $S(\mathrm{mV} / \mathrm{W})$ & 3.83 & 14.82 & 29.57 \\
\hline
\end{tabular}

responsivity of the Ni sample by at least $6 \%$ due to the nonlinear temperature dependence of electrical resistivity.

In the total result, the ratio of responsivity for the thin film samples, according to the experimental data, was determined as follows: $S_{\mathrm{TiNi}-1} / S_{\mathrm{Ni}}=3.9 \pm 0.3 ; S_{\mathrm{TiNi}-2} / S_{\mathrm{Ni}}=$ $7.7 \pm 0.5$. At the same time, $S_{\mathrm{TiNi}-2} / S_{\mathrm{TiNi}-1}=2.00 \pm 0.14$. This difference in responsivity (of the titanium nickelide films) results from differences in processing that manifested itself on the physical properties of the alloy. This assertion is supported by the fact that $\alpha_{\mathrm{TiNi}-2} / \alpha_{\mathrm{TiNi}-1}=1.29 \pm 0.07$, and the thermal mass of the TiNi-2 sample compared with that of the TiNi-1 sample was at least 50\% less according to eq. (5). In our opinion, the calculated responsivity of the titanium nickelide sample was not achieved because the processing methodologies that were employed in producing that sample failed to produce a combination of the known physical material properties presented in Table 1.

However, the qualitative estimation of output noise equivalent power (NEP) ratio for $\mathrm{Ni}$ and TiNi-2 samples has shown that under our experimental conditions, $N E P_{\mathrm{Ni}} /$ $N E P_{\mathrm{TiNi}-2}>7$.

\section{Conclusions}

For the first time, results obtained in a study of the use of thin films constructed from titanium nickelide as the sensitive element in a bolometer are presented. The characteristics of the films were studied in the temperature range in which the equiatomic TiNi was in the single-phase low-temperature state. We found that the sensitivity of the TiNi sample produced by one of the previously described processing methodologies is at least 7 times larger than that of the Ni sample (overall dimensions and experimental conditions being essentially equal). We also observed that relevant parameters of the titanium nickelide film depend on the preliminary thermomechanical treatment of the alloy. During the experiment, the TiNi thin films demonstrated high stability of operation inherent in metal bolometers. In the future, we plan to optimize the component composition of the titanium nickelide alloy along with processing that is used in the production of thin films. Future work will include the measurement of parameters for the samples in the two-phase region characterized by higher TCR (several times higher) than that in the single-phase low-temperature state, which will allow us to obtain better results. 


\section{Acknowledgements}

The authors would like to thank Professor Yu. M. Andreev for helpful comments.

\section{References}

1 P. L. Richards: J. Appl. Phys. 76 (1994) 1.

2 K. A. Lee, Y. Guo, P. A. Stimson, K. A. Potter, J. Chiao and D. B. Rutledge: IEEE Trans Antennas Propag. 39 (1991) 425.

3 A. D. Oliver and K. D. Wise: Sens. Actuators, A 73 (1999) 222.

4 A. Rogalski: Acta Phys. Pol. A. 116 (2009) 389.

5 V. Brailovski, S. Prokoshkin, P. Terriault and F. Trochu: Shape Memory Alloys: Fundamentals, Modeling and Applications (ETS Publ, Montreal, 2003) p. 851.

6 M. V. S. Ramakrishna, G. Karunasiri, P. Neuzil, U. Sridhar and W. J. Zeng: Sens. Actuators, A 79 (2000) 122.

7 H. G. Heard: Laser Parameter Measurements Handbook (Wiley, New York, 1968) p. 489.

8 L. Boarino, E. Monticone, G. Amato, G. Lérondel, R. Steni, G. Benedetto, A. M. Rossi, V. Lacquaniti, R. Spagnolo, V. Lysenko and A. Dittmar: Microelectron. J. 30 (1999) 1149.

9 V. Erofeev and P. Vybornov: Russian Patent No. 2345334 (2009). 